\title{
Influence of the Non-Linearity of the Aerodynamic Coefficients on the Skewness of the Buffeting Drag Force
}

\author{
Vincent Denoël $^{*}{ }^{, 1)}$, Hervé Degée ${ }^{1)}$ \\ 1) Department of Material mechanics and Structures, University of Liège, Belgium
}

\begin{abstract}
This paper is devoted to the non linear quasi-steady aerodynamic loading. A linear approximation is often used to compute the response of structures to buffeting forces. Some researchers have however shown that it is possible to account for the non linearity of this loading. This non linearity can come (i) from the squared velocity or (ii) from the shape of the aerodynamic coefficients (as functions of the wind angle of attack).

In this paper, it is shown that this second origin can have significant implications on the design of the structure, particularly when the non linearity of the aerodynamic coefficient is important or when the transverse turbulence is important.
\end{abstract}

\section{INTRODUCTION}

Wind loads acting on bluff bodies like bridge decks are complex functions of the components of the turbulence and of the structural displacements and velocities. In order to simplify the representation of these loads, approached models are generally considered. Since a convenient linear approximation gives accurate results in many cases, such a model has been widely used during the last decades (e.g. Davenport, 1962, Simiu and Scanlan, 1978). In its most general formulation, this linear model consists in decomposing the wind loads in three terms: (i) the static wind loading, (ii) the self-excited forces and (iii) the buffeting forces:

$$
\begin{aligned}
& F_{D}(t)=F_{D_{s}}(t)+F_{D_{s e}}(t)+F_{D_{b}}(t) \\
& F_{L}(t)=F_{L_{s}}(t)+F_{L_{s e}}(t)+F_{L_{b}}(t) \\
& F_{M}(t)=F_{M_{s}}(t)+F_{M_{s e}}(t)+F_{M_{b}}(t)
\end{aligned}
$$

where $F_{D}(t), F_{L}(t)$ and $F_{M}(t)$ represent respectively the drag and lift forces and the pitching moment. This general definition of the loading involves the well-known flutter derivatives and aerodynamic transfer functions (e.g. Chen and Kareem, 2002). After having 
measured these functions in wind tunnel experiments, the dynamic response and stability studies of the whole structure can be realized.

As a particular case of this linear approximation model, the linear quasi-steady theory (Fig. 1) provides very particular approximations of the flutter derivatives and of the transfer functions in terms of the usual aerodynamic coefficients $\left(C_{D}, C_{L}, C_{M}\right)$ and their derivatives with respect to the angle of attack $\left(C_{D}^{\prime}, C_{L}^{\prime}, C_{M}^{\prime}\right)$. Even if this model is limited because of its inability to represent transient frequency dependent forces, it can however represent correctly the low frequency motions of the structure.

Furthermore this linear quasi-steady theory is also a particular case of another more general model: the (non linear) quasi-steady theory. Even if it is also limited to low frequency motions, this theory presents however the advantage to give a non linear model for the wind loading. It is thus interesting in the sense that these non linear effects bring new physical phenomena that can't be enlightened with the usual linear models.

This paper focuses on some of these phenomena (mainly on the effects of non-linearity of the aerodynamic coefficients) and on the way to account for them in numerical simulations.

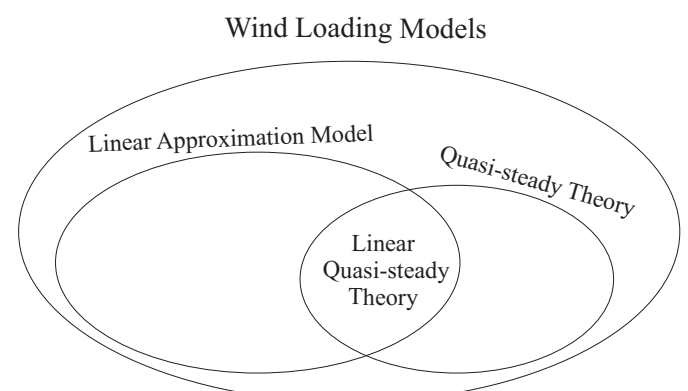

Fig. 1. Schematic view of wind loading models

\section{QUASI-STEADY FORMULATION OF THE WIND LOADING}

In order to clarify the context, a short recall of the usual linear quasi-steady theory is presented first.

The aerodynamic coefficients of a bridge deck are determined by measuring the aerodynamic forces $\left(F_{D}, F_{L}, F_{M}\right)$ acting on a fixed section placed in a wind tunnel:

$$
C_{D}=\frac{F_{D}}{\frac{1}{2} \rho B V^{2}} \quad ; \quad C_{L}=\frac{F_{L}}{\frac{1}{2} \rho B V^{2}} \quad ; \quad C_{M}=\frac{F_{M}}{\frac{1}{2} \rho B^{2} V^{2}}
$$

where $\rho, B$ and $V$ represent respectively the air density, the width of the deck and the constant wind velocity used for the experiment. 


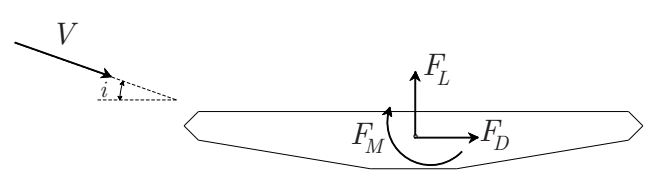

Fig. 2. Aerodynamic forces (drag, lift, moment)

For the range of wind velocities considered in practical applications (high Reynolds number), these aerodynamic coefficients can be considered to be independent of the wind velocity. On the other hand these coefficients are very dependent on the angle of attack $i$ of the wind with respect to the bridge deck. This is illustrated in Fig. 3 which represents drag coefficients of some famous European bridges. Any of these coefficients is indeed a nonlinear function of the angle of attack. For a convenient comparison, dotted lines represent tangents at the origin.

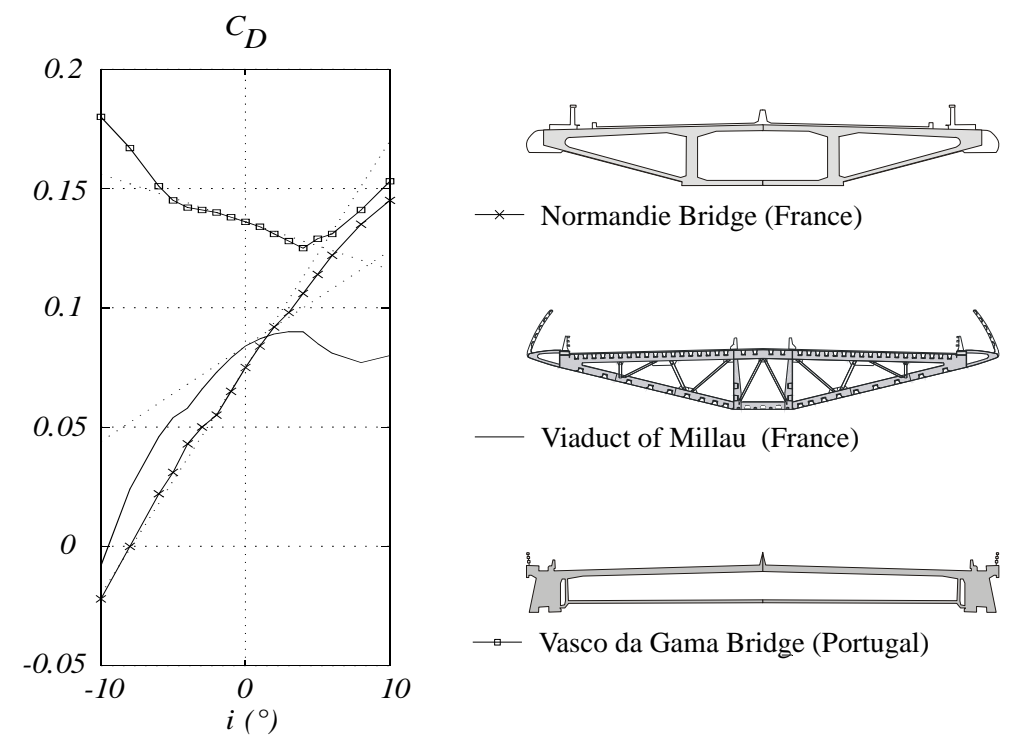

Fig. 3. Examples of drag coefficients (Cremona, 2002)

Provided the displacements of the structure are slow and their amplitudes remain small, Eq. (2) may be used to estimate the buffeting forces acting on a bridge deck. The subsequent developments can be done similarly for the drag and lift forces as well as the pitching moment. For the sake of brevity in the notations, drag forces only are studied in the following. The developments remain however also valid for the other aerodynamic coefficients.

The time varying formulation for the drag force is:

$$
F_{D}(t)=\frac{1}{2} C_{D}[i(t)] \rho B V^{2}(t)
$$

where the aerodynamic coefficient $C_{D}$ is now time dependent (trough the angle of attack $i$ ) and where the wind velocity is also time dependent since it depends on the mean wind speed $U$ and on the turbulence components $u(t)$ and $v(t)$ (Fig. 4). 
In order to partially account for a fluid-structure interaction, relative values must be considered for the wind angle of attack and the wind velocity. With notations of Fig. 4, these quantities can be expressed by:

$$
\begin{aligned}
& i(t)=\operatorname{ArcTan}\left(\frac{v(t)-h(t)}{U+u(t)-p(t)}\right)-\alpha(t) \\
& V^{2}(t)=(U+u(t)-p(t))^{2}+(v(t)-h(t))^{2}
\end{aligned}
$$

where the upper dot denotes a time derivative.

Introducing Eqs. (4) into Eq. (3) gives the non linear quasi-steady expression of the wind loading. As introduced before, it can be seen that this expression is a complex function of the components of the turbulence and the motion of the structure.

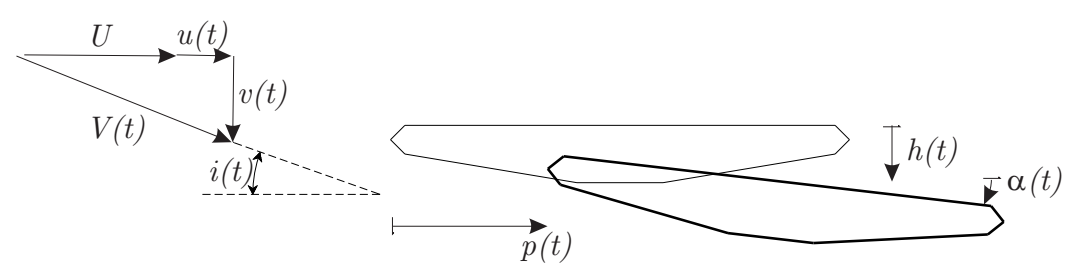

Fig. 4. Displacements of the structure

The components of turbulence are known in a probabilistic way only (Simiu, 1974). The most common methods that can be used to compute the dynamic response of a bridge are therefore:

- A stochastic analysis procedure (see e.g. Clough and Penzien, 1993), which is based, in its most basic formulation, on the computation of the power spectral density (PSD) of the response of the bridge as a function of the PSD of the turbulence components and of the mechanical and aerodynamic properties of the bridge;

- To use Monte Carlo simulations; this consists in generating wind histories and solving several times a deterministic problem by means of step-by-step analyses. This method allows accounting for the complete non linear expression but is rather slow since many generations are needed for a good accuracy. Thus this method should be used essentially to check results obtained by a stochastic analysis.

\section{Linearization of the aerodynamic loading}

A couple of decades ago, the field of application of stochastic procedures was limited by the abilities of computation means: probabilistic properties of structural response could thus be determined up to the second order only (variances). Therefore the response was generally considered to be Gaussian.

As the components of the turbulence can be considered as Gaussian, the classical procedure consists in simplifying the expression of the loading to a linear function of the turbulence. The loading is then Gaussian, and hence, if the structure can be assumed to behave linearly, the structural response is Gaussian too.

After having linearized Eqs. (4), introduced the resulting equations into Eq. (3), replaced the exact expression of aerodynamic coefficient by a linear approximation, and finally 
removed the subsequent quadratic terms, the linear quasi-steady formulation can be obtained (Cremona, 2002):

$$
F_{D}(t)=\frac{1}{2} \rho B U^{2}\left[C_{D_{0}}-\left(2 C_{D_{0}} \frac{\square(t)}{U}+C_{D_{0}}^{\prime} \frac{\square(t)}{U}+C_{D_{0}}^{\prime} \alpha(t)\right)+\left(2 C_{D_{0}} \frac{u(t)}{U}+C_{D_{0}}^{\prime} \frac{v(t)}{U}\right)\right]
$$

It can be seen that this expression is a particular case of Eqs. (1) since each term is a linear expression of the displacements (or velocities) of the structure or of the components of the turbulence. Since the vertical structural velocity $(h(t))$ and the rotation of the deck $(\alpha(t))$ are present in this expression of the drag force, Eq. (5) shows that a coupling between vertical, horizontal and torsional motions could exist.

\section{Higher degree polynomial approximation of the aerodynamic loading}

Nowadays more complex theories allow accounting for a non linear loading provided it is expressed as a polynomial approximation of the actual loading. Instead of linearizing Eq. (3), a higher order polynomial approximation can be used:

$$
F_{D}(t)=\frac{1}{2} \rho B U^{2}\left[-2 C_{D_{0}} \frac{\stackrel{p}{p}(t)}{U}-C_{D_{0}}^{\prime} \frac{\square(t)}{U}-C_{D_{0}}^{\prime} \alpha(t)+\sum_{l=0}^{\infty} \sum_{k=0}^{\infty} F_{k l} \frac{u^{l}(t) v^{k}(t)}{U^{l+k}}\right]
$$

\begin{tabular}{|c|c|c|c|c|c|}
\hline$F_{k l}$ & $K=0$ & $k=1$ & $k=2$ & $K=3$ & $K=4$ \\
\hline$l=0$ & $C_{D_{0}}$ & $C_{D_{0}}^{\prime}$ & $C_{D_{0}}+\frac{C_{D_{0}}^{\prime \prime}}{2}$ & $\frac{2 C_{D_{0}}^{\prime}}{3}+\frac{C_{D_{0}}^{\prime \prime \prime}}{6}$ & $\frac{C_{D_{0}}^{\prime \prime}}{6}+\frac{C_{D_{0}}^{\prime \prime \prime \prime}}{24}$ \\
\hline$l=1$ & $2 C_{D_{0}}$ & $C_{D_{0}}^{\prime}$ & 0 & $\frac{-2 C_{D_{0}}^{\prime}}{3}-\frac{C_{D_{0}}^{\prime \prime \prime}}{6}$ & $\frac{-C_{D_{0}}^{\prime \prime}}{3}-\frac{C_{D_{0}}^{\prime \prime \prime \prime}}{12}$ \\
\hline$l \approx 2$ & $C_{D_{0}}$ & 0 & 0 & $\frac{2 C_{D_{0}}^{\prime}}{3}+\frac{C_{D_{0}}^{\prime \prime \prime}}{6}$ & $\frac{C_{D_{0}}^{\prime \prime}}{2}+\frac{C_{D_{0}}^{\prime \prime \prime \prime}}{8}$ \\
\hline$I=3$ & 0 & 0 & 0 & $\frac{-2 C_{D_{0}}^{\prime}}{3}-\frac{C_{D_{0}}^{\prime \prime \prime}}{6}$ & $\frac{-2 C_{D_{0}}^{\prime \prime}}{3}-\frac{C_{D_{0}}^{\prime \prime \prime \prime}}{6}$ \\
\hline$I \approx 4$ & 0 & 0 & 0 & $\frac{2 C_{D_{0}}^{\prime}}{3}+\frac{C_{D_{0}}^{\prime \prime \prime}}{6}$ & $\frac{5 C_{D_{0}}^{\prime \prime}}{6}+\frac{5 C_{D_{0}}^{\prime \prime \prime \prime}}{24}$ \\
\hline
\end{tabular}

Table 1: Values of the parameters $F_{k l}$ (Denoel, 2005)

This expression is however obtained by neglecting non linear terms of the structural motions. Non-linear components of buffeting forces only are kept in this approach. The values of the coefficients $F_{k l}$ in Eq. (6) are given in Table 1 for the first values of $\boldsymbol{K}$ and $\boldsymbol{z}$. These coefficients are expressed in terms of the first four derivatives of $C_{D}(i)$ with respect to the angle of attack; these derivatives are defined by: 


$$
C_{D}(i)=C_{D_{0}}+i C_{D_{0}}^{\prime}+\frac{i^{2}}{2 !} C_{D_{0}}^{\prime \prime}+\frac{i^{3}}{3 !} C_{D_{0}}^{\prime \prime \prime}+\frac{i^{4}}{4 !} C_{D_{0}}^{\prime \prime \prime \prime}+\ldots
$$

It can be seen that the curvature of the aerodynamic coefficient $C_{D_{0}}^{\prime \prime}$ is present in a second order term of the loading $(\boldsymbol{K}=\boldsymbol{Z}, \boldsymbol{L}=\mathbf{0})$. It can be also checked that Eq. (5) is a particular case of Eq. (6).

In Eq. (6) the terms containing the derivatives of the bridge deck are related to the aerodynamic damping. Because they have already been widely studied and since the main aim of the paper concerns the effects of the non linear buffeting forces, these terms will be neglected in the following.

Since it is able to include any order of non linearity, the non linear model represented by Eq. (6) is very general. In order to enlighten in closed form the most important influences of the non linear loading, this paper focuses on the second order approximation of Eq. (6) only:

$$
\frac{F_{D}(t)}{\frac{1}{2} \rho B U^{2}}=C_{D_{0}}+2 C_{D_{0}} \frac{u(t)}{U}+C_{D_{0}}^{\prime} \frac{v(t)}{U}+C_{D_{0}} \frac{u^{2}(t)}{U^{2}}+C_{D_{0}}^{\prime} \frac{u(t) v(t)}{U^{2}}+\left(C_{D_{0}}+\frac{C_{D_{0}}^{\prime \prime}}{2}\right) \frac{v^{2}(t)}{U^{2}}
$$

The non linearity of the aerodynamic coefficient can therefore be taken into account up to its second order derivative only. Let us also consider the habitual hypothesis of Gaussian uncorrelated components of the turbulence $u(t)$ and $v(t)$ :

$$
\begin{gathered}
E[u(t)]=0 ; \quad E\left[u^{2}(t)\right]=\sigma_{u}^{2} \\
E[v(t)]=0 ; \quad E\left[v^{2}(t)\right]=\sigma_{v}^{2} \\
E[u(t) v(t)]=0 ;
\end{gathered}
$$

With these notations and assumptions, the statistical properties of the loading can be computed up to the third order: 


$$
\begin{aligned}
& \frac{\bar{F}_{D}(t)}{\frac{1}{2} \rho B U^{2}}=C_{D_{0}}+C_{D_{0}} \frac{\sigma_{u}^{2}}{U^{2}}+\left(C_{D_{0}}+\frac{C_{D_{0}}^{\prime \prime}}{2}\right) \frac{\sigma_{v}^{2}}{U^{2}} \\
& \frac{\sigma_{F_{D}}^{2}}{\left(\frac{1}{2} \rho B U^{2}\right)^{2}}=\left(4 C_{D_{0}}^{2} \frac{\sigma_{u}^{2}}{U^{2}}+C_{D_{0}}^{\prime 2} \frac{\sigma_{v}^{2}}{U^{2}}\right)+\left(2 C_{D_{0}}^{2} \frac{\sigma_{u}^{4}}{U^{4}}+C_{D_{0}}^{\prime 2} \frac{\sigma_{u}^{2} \sigma_{v}^{2}}{U^{4}}+2 C_{D_{0}}^{2} \frac{\sigma_{v}^{4}}{U^{4}}\right) \\
& +C_{D_{0}}^{\prime \prime}\left(2 C_{D_{0}}+\frac{C_{D_{0}}^{\prime \prime}}{2}\right) \frac{\sigma_{v}^{4}}{U^{4}} \\
& \frac{m_{F_{D}}^{(3)}}{\left(\frac{1}{2} \rho B U^{2}\right)^{3}}=3\left(8 C_{D_{0}}^{3} \frac{\sigma_{u}^{4}}{U^{4}}+4 C_{D_{0}} C_{D_{0}}^{\prime 2} \frac{\sigma_{u}^{2} \sigma_{v}^{2}}{U^{4}}+C_{D_{0}}^{\prime 2}\left(2 C_{D_{0}}+C_{D_{0}}^{\prime \prime}\right) \frac{\sigma_{v}^{4}}{U^{4}}\right)+ \\
& +\left(8 C_{D_{0}}^{3} \frac{\sigma_{u}^{6}}{U^{6}}+6 C_{D_{0}} C_{D_{0}}^{\prime 2} \frac{\sigma_{u}^{4} \sigma_{v}^{2}}{U^{6}}+3 C_{D_{0}}^{\prime 2}\left(2 C_{D_{0}}+C_{D_{0}}^{\prime \prime}\right) \frac{\sigma_{u}^{2} \sigma_{v}^{4}}{U^{6}}\right) \\
& +\left(2 C_{D_{0}}+C_{D_{0}}^{\prime \prime}\right)^{3} \frac{\sigma_{v}^{6}}{U^{6}}
\end{aligned}
$$

In the following these relations will be considered as reference values. Fig. 5 illustrates these first three non-dimensional statistical moments for several values of the wind intensities $I_{u}=\sigma_{u} / U$ and $I_{v}=\sigma_{v} / U$. These are computed for the drag coefficients of the bridges given in Fig. 3. The coefficients of the quadratic approximation are obtained by a least square fit with Gaussian weight distribution:

Normandy Bridge : $\quad C_{D_{0}}=0.0748 \quad ; \quad C_{D_{0}}^{\prime}=0.476 \quad ; \quad C_{D_{0}}^{\prime \prime}=-0.66$

$$
\text { Millau Viaduct : } \quad C_{D_{0}}=0.0830 \quad ; \quad C_{D_{0}}^{\prime}=0.202 \quad ; \quad C_{D_{0}}^{\prime \prime}=-3.40
$$

$$
\text { Vasco da Gama Bridge : } C_{D_{0}}=0.1337 \quad ; \quad C_{D_{0}}^{\prime}=-0.103 \quad ; \quad C_{D_{0}}^{\prime \prime}=1.54
$$

It can be proved that the actual statistical distribution of the angle of attack is almost Gaussian when both wind intensities are almost the same (Denoel, 2005), which justifies the use of a Gaussian weighting function. 



Fig. 5. "Exact" statistical characteristics of the loading as a function of the wind intensities

From these reference values of the statistical moments of the loading, two particular approximations can be derived.

\section{First approximation : linearization of the aerodynamic coefficient $\left(C_{D_{0}}^{\prime \prime}=0\right)$}

As a first approximation, it could be supposed that the aerodynamic coefficient is linear $\left(C_{D_{0}}^{\prime \prime}=0\right)$. Since non linear terms coming from the expression of the squared velocity are kept, the subsequent expression of loading remains non-linear and hence non Gaussian (see Eq. (8), in which $C_{D_{0}}^{\prime \prime}=0$ ) . The approached statistical values obtained under this hypothesis are determined by imposing $C_{D_{0}}^{\prime \prime}=0$ in Eqs. (10). They are represented in Fig. 6. 

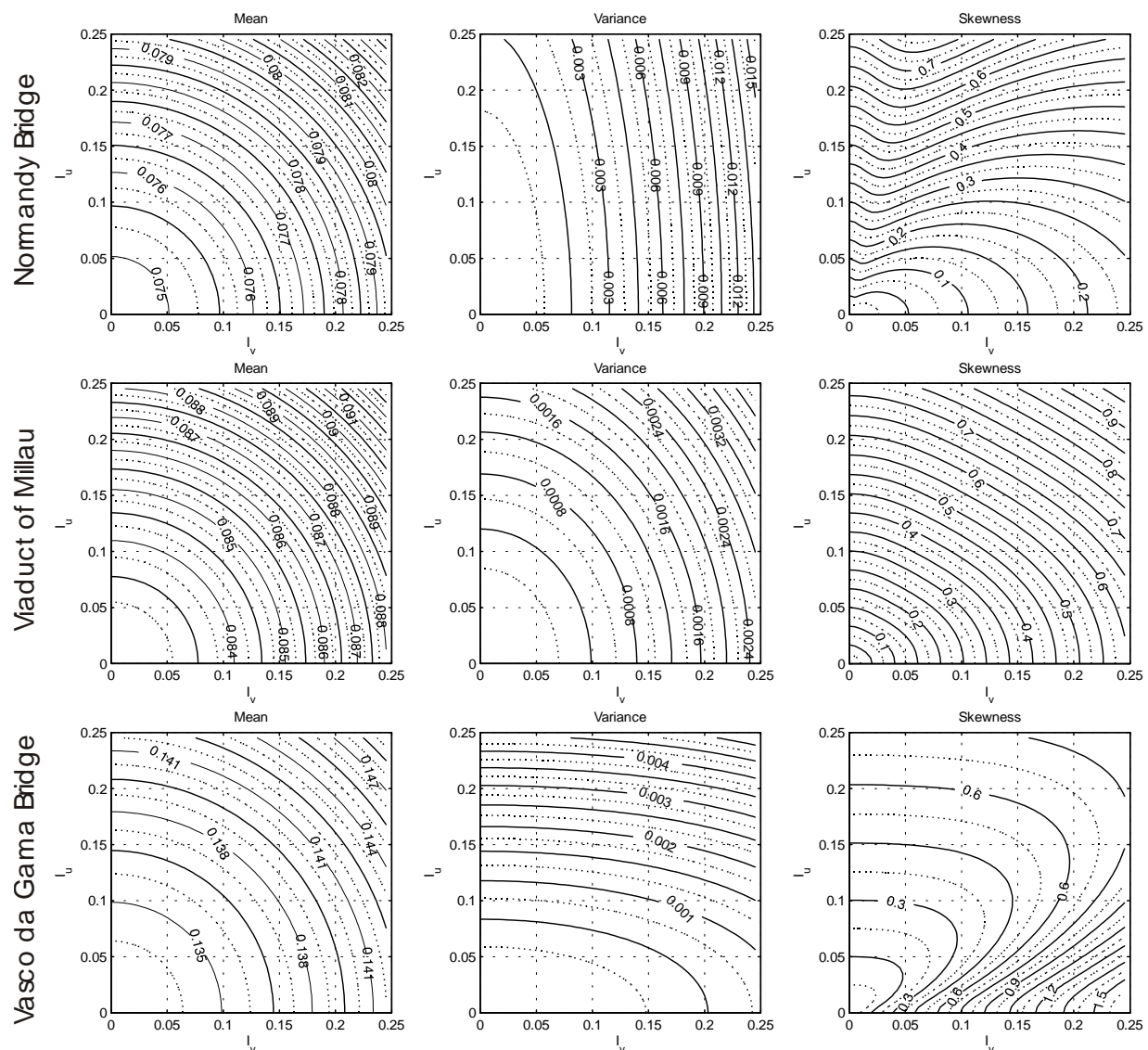

Fig. 6. Approached statistical characteristics of the loading as a function of wind intensities

The first developments concerning the non Gaussianity of the aerodynamic loading correspond to this hypothesis. Many authors (Grigoriu, 1986, Benfratello, 1996, Kareem, 1998, Gusella, 2000, Floris, 2002) have studied the effects of the wind intensity on the skewness of the loading. These early developments were based on a 1-D turbulence field $\left(I_{v}=0\right)$. In this case, the comparison of Figs. 5 and 6 shows that the exact and approached means, variances and skewnesses are exactly the same, as the values along the vertical axis only have to be compared. From a theoretical point of view, this indicates that the aerodynamic coefficients can be linearized provided the transverse wind intensity $I_{v}$ is equal to zero.

In practical applications, the transverse intensity $I_{v}$ is however generally not equal to zero and the vertical axis of Figs. 5 and 6 is not sufficient anymore to represent the actual statistical moments. The comparison of both figures shows that a small transverse intensity can modify drastically the values of the statistical moments. For example, for $I_{u}=10 \%$ and $I_{v}=10 \%$, the exact and approached non dimensional variances of the drag force on the Viaduct of Millau are respectively equal to $12.1 E-4$ and $6.9 E-4$. The originality of the paper lies in this observation and an important contribution is to provide statistical moments up to the third order, in a 2-D flow and accounting for the non linearity of the aerodynamic coefficients.

Regarding the skewness coefficient, the significantly different patterns depicted in Figs. 5 and 6 show that it is considerably affected by the non linearity of the aerodynamic coefficient. 
Furthermore, Fig. 5 exhibits negative skewness zones (for large $I_{v}$ ) that can not be explained with the approached model (Fig. 6). Consequently, the non linearity of the aerodynamic coefficient $\left(C_{D_{0}}^{\prime \prime} \neq 0\right)$ can turn the positively-skewed probability density function into a negatively-skewed one. In terms of extreme values, this may have very important consequences.

It is interesting to notice that the skewness coefficient associated to Normandy Bridge is significant despite the apparent linearity of its drag coefficient (Fig. 3).

\section{Second approximation : linearization of the aerodynamic loading}

As a second approximation, the equations of the linear quasi-steady loading could be derived. In this method, linear terms of the loading only must be considered: the first three terms of Eq. (8) only are thus kept. The non dimensional statistical characteristics of the loading are now much simpler than Eqs. (10):

$$
\frac{\bar{F}_{D}(t)}{\frac{1}{2} \rho B U^{2}}=C_{D_{0}} \quad ; \quad \frac{\sigma_{F_{D}}^{2}}{\left(\frac{1}{2} \rho B U^{2}\right)^{2}}=4 C_{D_{0}}^{2} \frac{\sigma_{u}^{2}}{U^{2}}+C_{D_{0}}^{\prime 2} \frac{\sigma_{v}^{2}}{U^{2}} \quad ; \quad \frac{m_{F_{D}}^{(3)}}{\left(\frac{1}{2} \rho B U^{2}\right)^{3}}=0
$$

Exactly as for the statistical moments given in Eq. (10), these values are obtained thanks to the theory of probabilities.
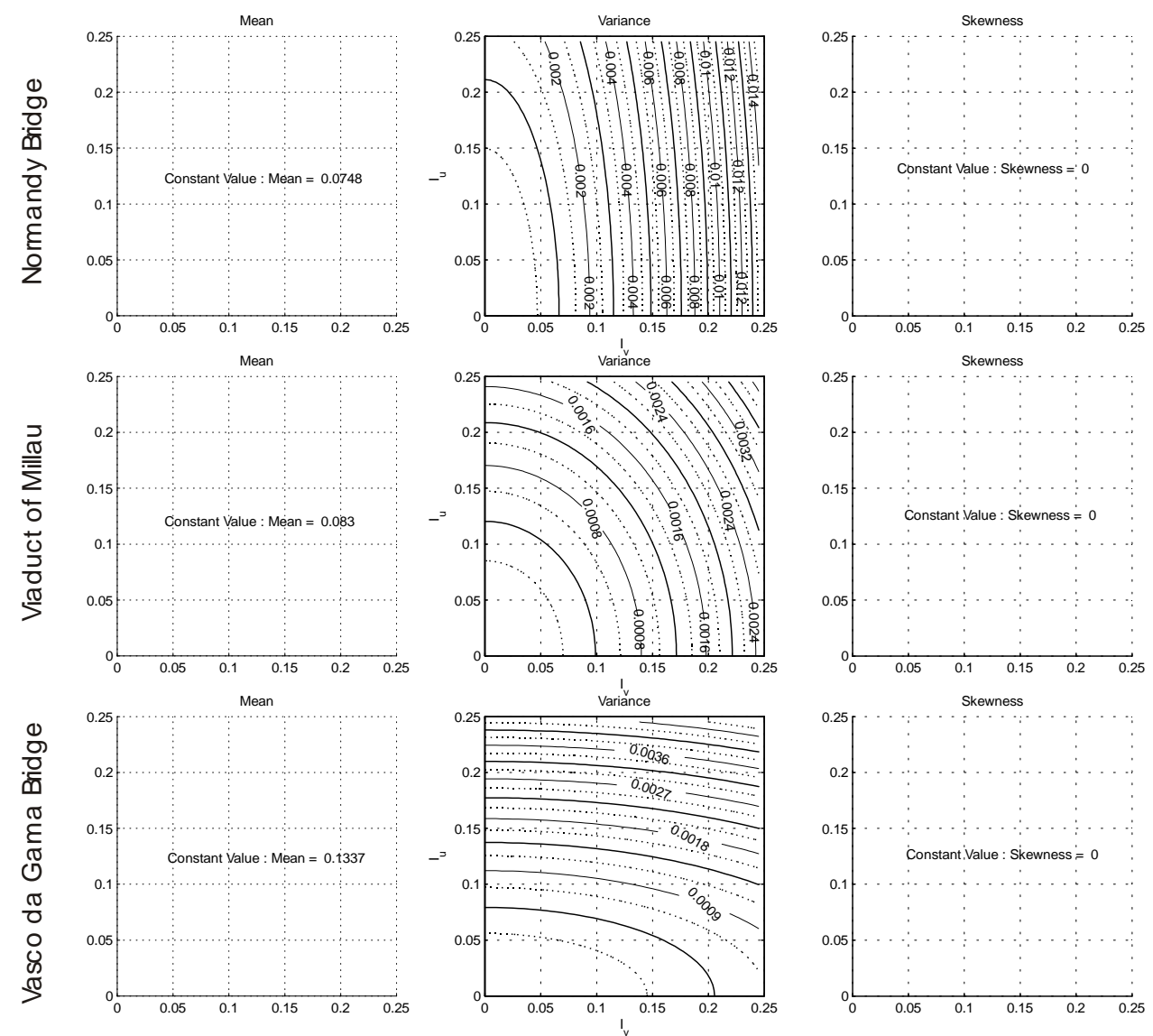
Fig. 7. Approached statistical characteristics of the loading as a function of wind intensities

They are plotted in Fig. 7. It can be checked that this method provides inaccurate results, even on the vertical axis. Furthermore since the loading is now Gaussian, this method gives a skewness coefficient obviously equal to zero.

\section{RESPONSE OF STRUCTURES TO NON GAUSSIAN LOADINGS}

Even if the theoretical background concerning the study of the dynamic response of structures subjected to non-Gaussian loading is quite old (Lutes and Hu, 1986, Soize, 1978), recent researches have led to new ways of applying this theory in practical applications. Two major philosophies can be distinguished:

- Third and fourth order characteristics can be represented by bispectra and trispectra, similarly as second order characteristics can be represented by PSDs (Gurley et al., 1997, Kareem, 1998, Spanos, 1983);

- Another possibility consists in solving a particular set of equations (the moment equations) derived from the Fokker-Planck-Kolmogorov equations (Di Paola, 1990). The main disadvantage of this method is that the force must be represented by a Markov chain; but when this condition is fulfilled, this second method is much faster that the first one since probabilistic characteristics of the response can be estimated by solving a simple set of algebraic equations.

Amongst both of them, this paper is based on the first one. The theoretical basis of this method is not presented in the paper but can be found in the literature (see e.g. Gurley et al., 1997).

The method is here applied to an interesting academic application: the study of a single degree of freedom system subjected to the simplest quadratic loading:

$$
\mathbb{x}(t)+2 \xi \varpi x(t)+\varpi^{2} x(t)=\gamma\left(U^{2}+2 U u(t)+u^{2}(t)\right)
$$

where $\xi$ and $\varpi$ represent the structural characteristics, $\gamma=\rho C_{D} B / 2$ is supposed to be constant and $u(t)$ represents a zero-mean Gaussian and Ornstein-Uhlenbeck process whose PSD is given by:

$$
S_{u}(\omega)=\frac{a}{\pi} \frac{\sigma_{u}^{2}}{a^{2}+\omega^{2}}
$$

where $\sigma_{u}^{2}=I_{u}^{2} U^{2}$ is the variance of the process, $I_{u}$ is the turbulence intensity and $a$ is a frequency-shaping parameter.

This academic application is worthy of note because it can be used to estimate the effects of the non linearity of the quadratic loading in a 2-D flow (Eq. (8)). This is discussed in the next section. 


\section{Second order response}

Even if the loading is non linear and hence non Gaussian, its PSD $\left(S_{F}(\omega)\right)$ can be expressed in terms of the PSD of the turbulence $\left(S_{u}(\omega)\right)$ (e.g. Floris, 2002). After multiplication by the transfer function of the system, the PSD of the response $\left(S_{u}(\omega)\right)$ can be obtained and finally, after integration along the frequencies, the variance of the displacement $\sigma_{x_{\text {quad }}}^{2}$ can be obtained. This is the "exact" variance. As a comparison, the variance of the response $\left(\sigma_{x_{\text {tin }}}^{2}\right)$ obtained by neglecting the non-linear terms of the loading can also be computed.

The ratio between these two variances is a measure of the influence of the quadratic term of the loading on the second order characterization of the structural motion. An approached (because it is valid for small turbulence intensities, namely smaller than 20\%) expression of this ratio is given by (Appendix A):

$$
\frac{\sigma_{x_{\text {quad }}}^{2}}{\sigma_{x_{\text {lin }}}^{2}} \approx \Psi\left(1+\frac{I_{u}^{2}}{2}\right)+(1-\Psi)\left(1+I_{u}^{2} \frac{1+(\varpi / a)^{2}}{4+(\varpi / a)^{2}}\right)
$$

where $\Psi$ represents the ratio between the quasi-static component and the total variance of the response.

Fig. 8 illustrates this ratio of variances in the most realistic case (which appears to be also the worst case), namely when $\varpi / a$ is much larger than unity.

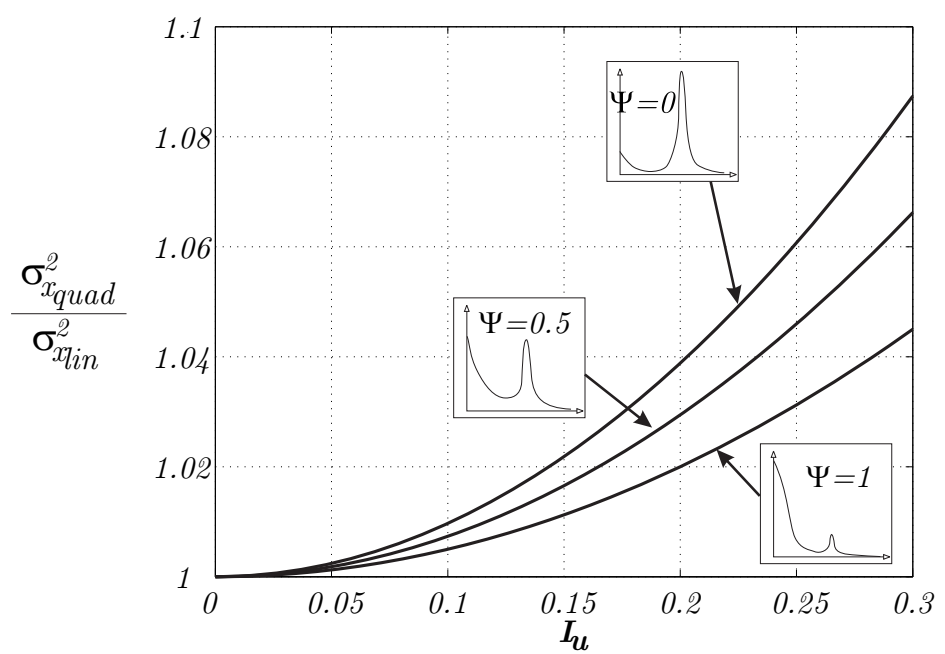

Fig. 8. Influence of the quadratic term of the loading on the variance of the displacement (as a function of the dispatching of energy between quasi-static and dynamic contributions)

This figure shows that the non linear term of the loading produces a more significant influence when the response of the structure is essentially dynamic and not quasi-static (i.e. when $\Psi=0)$. It can be seen that for usual values of the wind intensity $\left(I_{u} \square 15 \%\right.$ to $I_{u} \square 20 \%$ ), the quadratic term of the loading doesn't affect significantly the variance of the response. This observation should however be mitigated if the coefficient of $u^{2}(t)$ in the loading was larger than the coefficient of $U^{2}$. 
This figure shows also that the ratio of the variance of the response for $\Psi=0$ and $\Psi=1$ is equal to $\left(1+I_{u}^{2}\right) /\left(1+I_{u}^{2} / 2\right)$. Furthermore, for any $\Psi$ this ratio could be estimated by:

$$
f(\Psi)=\frac{\sigma_{x_{\text {quad }, \Psi}}^{2}}{\sigma_{x_{\text {quad }, Q S(\Psi=1)}}^{2}}=\frac{\Psi\left(1+\frac{I_{u}^{2}}{2}\right)+(1-\Psi)\left(1+I_{u}^{2}\right)}{1+\frac{I_{u}^{2}}{2}}=1+(1-\Psi) \frac{\frac{I_{u}^{2}}{2}}{1+\frac{I_{u}^{2}}{2}}
$$

This shows that the exact variance of the response under quadratic loading ${\sigma^{2}}_{x_{\text {quad }}}^{2}$ can be estimated by multiplying the quasi-static contribution of the variance by a simple function of $\Psi$ and $I_{u}$. Note that the function given in Eq. (16) is valid for an Orstein-Uhlenbeck loading process only.

In practical applications, the turbulence can very rarely be considered as an OrsteinUhlenbeck process; but let us suppose that the statistical moments of the loading have been computed following the developments of this section. This computation implies simple algebraic operations only.

If the structure is very stiff, its response is mainly quasi-static and, in this case, the variance of the structure is obtained by dividing the variance of the loading by the squared stiffness. In practical applications, the response is divided into quasi-static and dynamic components. In this more general case, Eq. (16) can be used to give an estimation of the complete variance. Indeed, the response including these two components can be estimated from the knowledge of the quasi-static counterpart only (under quadratic loading). Although the function given in Eq. 16 is valid for an Ornstein-Uhlenbeck process, it could be applied in good approximation to most usual PSD function of wind turbulence (Harris, Scanlan, Davenport, Eurocode 1),(Denoel, 2005). This approximation presents the advantage to give very fast results.

\section{Third order analysis}

Several authors (Soize, 1978, Gurley et al., 1997) have shown that the extreme values of non Gaussian processes depend of their higher order statistical characteristics, namely their skewness and kurtosis defined by:

$$
\gamma_{3}=\frac{m_{x}^{(3)}}{\sigma_{x}^{3}} \quad ; \quad \gamma_{4}=\frac{m_{x}^{(4)}}{\sigma_{x}^{4}}
$$

where $m_{x}^{(n)}=E\left[(x-\bar{x})^{n}\right]$ represents the $\mathrm{n}^{\text {th }}$ centred moment of the process $\boldsymbol{z}$.

Gurley et al. propose to compute the extreme values as if the process was Gaussian and then to multiply this first estimation by a correcting factor that accounts for the non Gaussianity (see Fig. 9). This factor is expressed as a function of the mean crossing rate (v) and of the duration of the observation $\mathrm{T}$. The correcting factor is of course equal to unity for a Gaussian process, for which $\gamma_{3}=0$ and $\gamma_{4}=3$. 

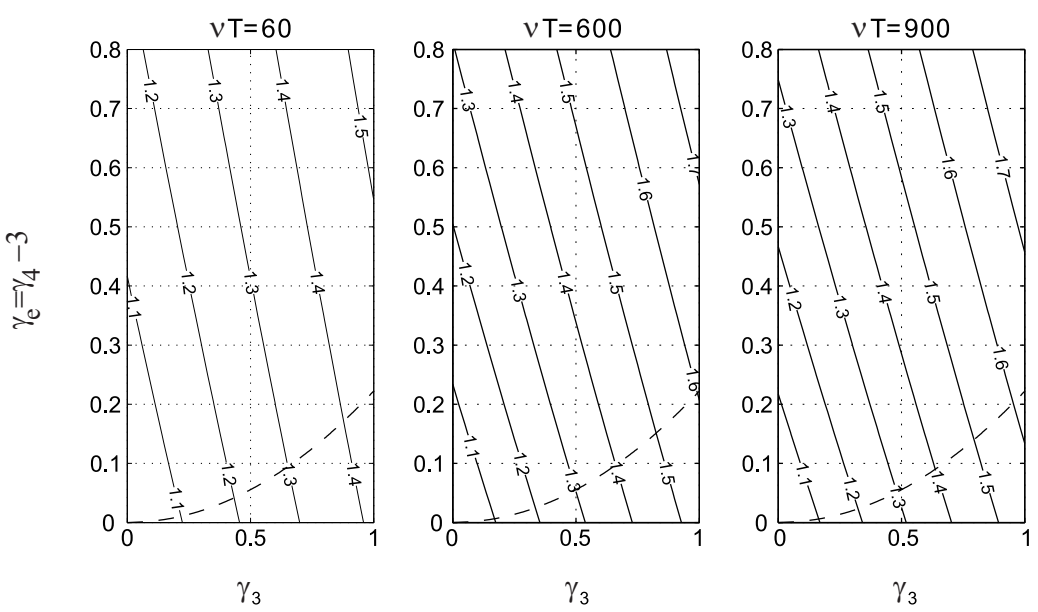

Fig. 9. Correcting factor proposed by Gurley et al. to account for the effect of non Gaussianity on extreme values.

If it is desired to estimate the effects of the non linearity of the loading on the extreme values of the displacement, it is thus necessary to compute higher order statistical characteristics of the response. Exactly as the variance can be obtained by integration of the PSD, the third centred moment can be estimated by integration of another mathematical quantity: the bispectrum. Since, in our developments, the loading (Eq. (8)) is considered as a polynomial form of a Gaussian process $\boldsymbol{u}(\boldsymbol{t})$, the bispectra of the loading and of the response can be determined in an analytical way.

If the non linear term of the loading was neglected, the response would be Gaussian and the skewness coefficient would be equal to zero. The ratio $\gamma_{3 x_{\text {quad }}} / \gamma_{3 x_{\text {lin }}}$ similar to the ratio used in the previous paragraph is thus meaningless.

Instead of this ratio it is thus chosen to evaluate the ratio of the skewness coefficient of the response to the skewness coefficient of the loading. Fig. 10 represents this ratio as a function of the damping coefficient, the circular frequencies and the parameter $\boldsymbol{a}$. These curves have been obtained in closed form (by analytical integration of the bispectrum of the response) without any other hypothesis. The resulting (heavy) relations are not provided in this paper but can be found in the literature (Denoel, 2005). This figure points out the convenient possibility to determine the skewness of the response as a function of the skewness of the loading. 


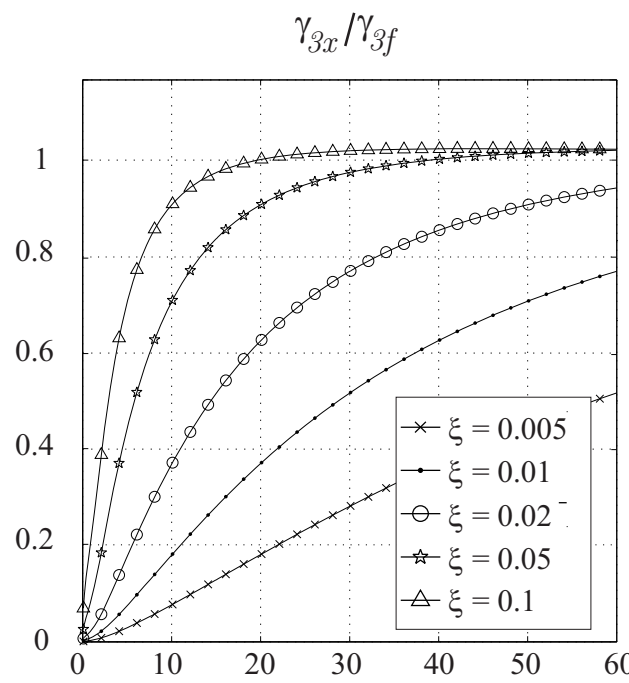

$\bar{\omega} / a$

Fig. 10. Importance of the skewness of the response as a function of the skewness of the loading, the damping coefficient and the eigen frequency (closed form)

It can be observed that:

- the skewness of the response is always smaller than the skewness of the loading;

- the skewness of the response is small for lightly damped or soft structures;

These observations can be justified by considering that the response of the structure is composed of two contributions: (i) the quasi-static contribution, shaped like the applied force and thus characterized by the same skewness coefficient, and (ii) the dynamic component, rather shaped like a Gaussian process. It seems thus obvious to obtain an intermediate skewness coefficient for the response, this coefficient being smaller for structures having an important dynamic contribution, i.e. for lightly damped and soft structures.

The analytical relations used to draw Fig. 10 can be useful in several applications. Let us imagine for instance that the actual PSD of the turbulence could be approached by an Ornstein-Uhlenbeck process (e.g. by fitting parameter a). Several authors (Muscolino, 1995, Floris, 2002) have proposed some formulations for this equivalence which consists in replacing the actual PSD by an Ornstein-Uhlenbeck one.

In the context of a fast estimation of the non Gaussianty of the response, Fig. 10 shows that the skewness coefficient of the loading could be used as a boundary value for the design of the structure. If the dynamic component of the response is important, its skewness coefficient can be significantly reduced and Fig. 10 can help giving a better approximation of it. This value can be simply estimated by the knowledge of:

- the skewness coefficient of the loading,

- the damping coefficient of the structure,

- the eigen frequency of the structure,

- an equivalent shaping factor $\boldsymbol{a}$

which is clearly much simpler than computing and integrating the exact bispectrum. 


\section{CONCLUSIONS}

This paper has presented two important features in the analysis of structures subjected to non Gaussian loadings.

The first one consists in the determination of the statistical characteristics of the loading. They are commonly established by supposing that the aerodynamic coefficients can be linearized. This linearization can lead to a significant inaccuracy on the statistical characteristics. The developments presented in this paper were limited to the second order derivative of the aerodynamic coefficients with respect to the angle of attack but the same reasoning could be easily extended to higher order polynomial approximations.

As a second step, it has been shown how the statistical characteristics of the loading could be used to estimate the statistical characteristics of the response. In this part, the heavy rigorous analysis method has been avoided and a simpler approach, based on simple and fast computations, has been retained. This effortless method can give estimations of the response that could be used, for instance, for a pre-design of a structure.

\section{ACKNOWLEDGMENTS}

The authors would like to acknowledge the Belgian National Fund for Scientific Research.

\section{APPENDIX}

Under the loading conditions of Eqs. (13) and (14) the PSD of the non linear loading force is given by:

$$
S_{F}(\omega)=\left(U^{2}\right)^{2} \frac{4 a}{\pi}\left(\frac{I_{u}^{2}}{a^{2}+\omega^{2}}+\frac{I_{u}^{4}}{4 a^{2}+\omega^{2}}\right)
$$

where the first and second terms in the brackets respectively come from the linear and the non linear counterparts of the loading. The traditional white noise approximation (Davenport, 1962) prescribes the estimation of the response as the sum of the background and the resonant components. This method can be applied to determine the response to the linear loading on the one hand and to the non linear one on the other hand. Their ratio is given by:

$$
\frac{\sigma_{x_{\text {quad }}}^{2}}{\sigma_{x_{\text {lin }}}^{2}}=\frac{\sigma_{x_{\text {quad }}, q s}^{2}+\sigma_{x_{\text {quad }}, d y}^{2}}{\sigma_{x_{\text {lin }}}^{2}}=\frac{\sigma_{x_{\text {quad }}, q s}^{2}}{\sigma_{x_{\text {lin }}, q s}^{2}} \underbrace{\frac{\sigma_{x_{\text {lin }}, q s}^{2}}{\sigma_{x_{\text {lin }}}^{2}}}_{\Psi}+\frac{\sigma_{x_{\text {quad }}, d y}^{2}}{\sigma_{x_{\text {lin }}, d y}^{2}} \underbrace{\frac{\sigma_{x_{\text {lin }}, d y}}{\sigma_{x_{\text {lin }}}^{2}}}_{1-\Psi}
$$

which is now a function of the quasi-static and dynamic contributions under the linear and non linear loading. Recalling that the quasi-static response is obtained by dividing the variance of the loading by the stiffness and that the dynamic response is expressed as a function of the PSD of the loading at the natural frequency, it is possible to write: 


$$
\begin{gathered}
\frac{\sigma_{x_{\text {quad }}, q s}^{2}}{\sigma_{x_{\text {lin }}, q s}^{2}}=\frac{\sigma_{F_{\text {quad }}}^{2}}{\sigma_{F_{\text {lin }}}^{2}}=\frac{4 I_{u}^{2}+2 I_{u}^{4}}{4 I_{u}^{2}}=1+\frac{I_{u}^{2}}{2} \\
\frac{\sigma_{x_{\text {quad }}, d y}^{2}}{\sigma_{x_{\text {lin }}, d y}^{2}}=\frac{S_{F_{\text {quad }}}^{2}(\varpi)}{S_{F_{\text {lin }}}^{2}(\varpi)}=\frac{\frac{I_{u}^{2}}{a^{2}+\varpi^{2}}+\frac{I_{u}^{4}}{4 a^{2}+\varpi^{2}}}{\frac{I_{u}^{2}}{a^{2}+\varpi^{2}}}=1+I_{u}^{2} \frac{1+(\varpi / a)^{2}}{4+(\varpi / a)^{2}}
\end{gathered}
$$

The introduction of Eqs. (A.3) and (A.4) into (A.2) is equivalent to Eq. (15). This relation is approached because it is based on the white noise approximation. A deeper investigation into this problem (Denoel, 2005) shows that this approximation is however suitable if the turbulence intensity is smaller than $20 \%$, which results in discrepancies smaller than $5 \%$.

\section{REFERENCES}

Benfratello S., Falsone G., Muscolino G. (1996), "Influence of the quadratic term in the along wind stochastic response of SDOF structures”. Engineering Structures, Vol. 18, 685695.

Chen X., Kareem A. (2002), "Advances in modelling of aerodynamic forces on bridge decks”, Journal of Engineering Mechanics, Vol 128(11), 1193-1250.

Clough R.W., Penzien J. (1993), Dynamics of Structures, Mc Graw-Hill : Civil Engineering series (second edition).

Cremona C., Foucriat J.C. (2002), Comportement au vent des ponts, Presses de l'Ecole Nationale des Ponts et Chaussées, Paris (in french).

Davenport A.G. (1962), "The response of slender line-like structures to a gusty wind", Proceedings of the Institute of Civil Engineers, Vol 23, 389-408.

Denoël V. (2005), “Application des méthodes d'analyse stochastique à l'étude des effets du vent sur les structures du génie civil”, PhD thesis, University of Liège, Belgium (in french).

Di Paola M., Muscolino G. (1990), "Differential moment equations for FE modelled structures with geometrical non-linearities”, International Journal of Non Linear Mechanics, Vol 25(4), 363-373.

Floris C., Valloni (2002), "Non Gaussian response to random wind pressures : a comparison of different approaches", European Conference on Structural Dynamics, Eurodyn 2002, Munich (pp 773-778).

Grigoriu M. (1986), "Response of linear systems to quadratic gaussian excitation", Journal of Engineering Mechanics ASCE Vol 112(6) 729-744.

Gurley K., Tognarelli A., Kareem A. (1997) "Analysis and simulation tools for wind engineering”, Probabilistic Engineering Mechanics, Vol 12(1), 9-31.

Gusella V., Materazzi A.L. (2000), "Non Gaussian along wind response analysis in time and frequency”, Engineering Structures Vol 22, 49-57. 
Kareem A., Tognarelli A., Gurley K.R. (1998), "Modeling and analysis of quadratic term in the wind effects on structures", Journal of Wind Engineering and Industrial Aerodynamics, Vol 74, 1101-1010.

Lutes L.D., Hu S.L. (1986) "Non normal stochastic response of linear systems”, Journal of Engineering Mechanics, ASCE, Vol 112, 127-141.

Muscolino G. (1995) "Linear systems excited by polynomial forms of non-Gaussian filtered processes", Probabilistic Engineering Mechanics, Vol 10, 35-44.

Simiu E. (1974), "Wind Spectra and Dynamic Alongwind Response”, Journal of Structural Division ASCE Vol 100, 1897-1910.

Simiu E., Scanlan R.H (1978), "Wind effects on structures", John Wiley \& Sons. First Edition.

Soize C. (1978), "Gust loading factors with non linear pressure terms", Journal of the structural division ASCE, Vol 104(6), 991-1007.

Spanos P.D. (1983), "Spectral moments calculation of linear system output", Journal of Applied Mechanics ASME, Vol 50, 901-903. 